\title{
Analyses on Poisson's Problem by Using a New Collocation Method*
}

\author{
Yong-Ming GUO ${ }^{* *}$, Koki ISOZAKI** and Shunpei KAMITANI** \\ ${ }^{* *}$ Graduate School of Science and Engineering, Kagoshima University, \\ 1-21-40 Korimoto, Kagoshima City, 890-0065 Japan \\ E-mail: guoy@mech.kagoshima-u.ac.jp
}

\begin{abstract}
In this paper, the three boundary value problems on Poisson's problem are analyzed by using a new meshless method that is called the over-range collocation method (ORCM). By introducing some collocation points, which are located at outside of domain of the analyzed body, unsatisfactory issue of the positivity conditions of boundary points can be avoided. Convergence studies in the numerical examples show that the ORCM possesses good convergence for both the unknown variables and their derivatives. Quite accurate numerical results calculated by both regular nodal models and irregular nodal models have been obtained.
\end{abstract}

Key words: Meshless Method, Collocation Method, Moving Least-Square Approximation, Positivity Conditions, Over-Range Points

\section{Introduction}

In most meshless techniques, complicated non-polynomial interpolation functions are used which render the integration of the weak form rather difficult. Failure to perform the integration accurately results in loss of accuracy and possibly stability of solution scheme. The integration of complicated non-polynomial interpolation function also costs much CPU time. The collocation method has no issues of the integration scheme, the integration accuracy and the integration CPU time. Several collocation methods based on different types of approximations or interpolations have been proposed in the literature. Onate et al. (1) have proposed a finite point method based on weighted least squares interpolations for the analyses of convective transport and fluid flow problems. Onate et al. ${ }^{(2)}$ have also proposed a residual stabilization procedure, adequate for the finite point method, and further extended the finite point method to the solution of the advective-convective transport equations as well as those governing the flow of compressible fluids. Aluru ${ }^{(3)}$ has presented a point collocation method based on reproducing kernel approximations for numerical solution partial differential equations with appropriate boundary conditions. Jin, Li and Aluru ${ }^{(4)}$ have shown the robustness of collocation meshless methods can be improved by ensuring that the positivity conditions are satisfied when constructing approximation functions and their derivatives. Boroomand, Tabatabaei and Onate ${ }^{(5)}$ have presented a stabilized version of the finite point method to eliminate the ill-conditioning effect due to directional arrangement of the points. Atluri, Liu and Han ${ }^{(6)}$ have presented a MLPG mixed collocation method by using the Dirac delta function as the test function in the MLPG method, and shown that the MPLG mixed collocation method is more efficient than the other MLPG implementations, including the MLPG finite volume method. Atluri, Liu and Han ${ }^{(7)}$ have proposed a finite difference method, within the framework of the MLPG 
approach, for solving solid mechanics problems. The MLPG mixed collocation method has also been successfully used in material orientation and topology optimization of anisotropic solids and structures ${ }^{(8)}$. Kosec and Sarler ${ }^{(9)}$ have explored the application of the mesh-free local radial basis function collocation method in solution of coupled heat transfer and fluid flow problems in Darcy porous media. Wu, Chiu and Wang ${ }^{(10)}$ have developed a mesh-free collocation method based on differential reproducing kernel approximations for the three-dimensional analysis of simply-supported, doubly curved functionally graded magneto-electro-elastic shells under the mechanical load, electric displacement and magnetic flux.

But, the roughness of the collocation methods is an issue especially when scattered and random points are used. To improve the robustness of the collocation methods, Jin, Li and Aluru $^{(4)}$ have proposed techniques, based on modification of weighting functions, to ensure satisfaction of positivity conditions when using a scattered set of points. For boundary points, however, the positivity conditions cannot be satisfied, obviously. In this paper, three boundary value problems on Poisson's equation with a quadratic source function are analyzed by using a new over-range collocation method (ORCM) ${ }^{(11)}$, in which by introducing some collocation points that are located at outside of domain of the analyzed body, unsatisfactory issue of the positivity conditions of boundary points in collocation methods can be avoided.

\section{Formulation of the ORCM}

\subsection{Collocation Scheme}

Let us assume a scalar problem governed by a partial differential equation:

$$
D(u)=b, \quad \text { in } \Omega
$$

with boundary conditions

$$
\begin{aligned}
& T(u)=t, \quad \text { on } \Gamma_{t} \\
& u-u_{c}=0, \quad \text { on } \Gamma_{u}
\end{aligned}
$$

to be satisfied in a domain $\Omega$ with boundary $\Gamma=\Gamma_{t} \cup \Gamma_{u}$, where $D$ and $T$ are appropriate differential operators, $u$ is the problem unknown function, $b$ and $t$ are external forces or sources acting over $\Omega$ and along $\Gamma_{t}$, respectively. $u_{c}$ is the assigned value of $u$ over $\Gamma_{u}$.

Consider taking some collocation points in $\Omega$, at which Eq. (1) is satisfied, and some collocation points on $\Gamma_{t}$, at which both Eq. (1) and Eq. (2) are satisfied, as well as some collocation points on $\Gamma_{u}$, at which both Eq. (1) and Eq. (3) are satisfied. Besides the collocation points over $\Omega$, let us assume other collocation points located at outside of $\Omega$ and call them over-range points ( see Fig. 1 ). At outside of $\Omega$, neither satisfaction of any governing partial differential equation nor satisfaction of boundary condition is needed. Therefore, no over-constrained condition is imposed into the boundary value problem. While the over-range points can be used in interpolating calculation of boundary points, so

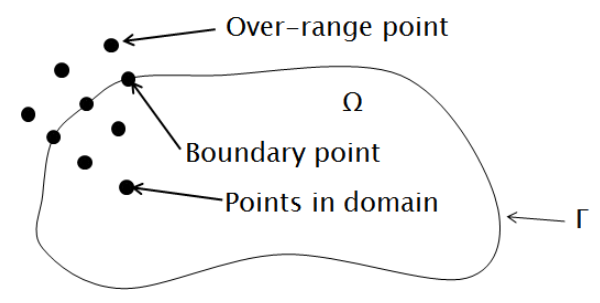

Fig. 1 Over-range points, boundary points and points in domain 
that the unsatisfactory issue of the positivity conditions of boundary points in collocation methods can be avoided.

Let us assume that the number of points in domain is $K_{d}$, the number of boundary points is $K_{b}$ and the number of over-range points is $K_{o}$, then the number of unknown variables is $2\left(K_{d}+K_{b}+K_{o}\right)$ for a 2-D problem. Because the number of equations of the ORCM is $2\left(K_{d}+K_{b}\right)+2 K_{b}$, by taking the same number of the equations with that of the unknown variables, we obtain that the number of the over-range points $K_{o}$ must be equal to the number of boundary points $K_{b}$.

\subsection{The MLS Approximation with Kronecker-Delta Property}

In the classical moving least-square (MLS) approximation, the shape functions have no Kronecker-delta property, so that the essential node condition cannot be imposed on boundaries. In this paper, a modified MLS approximation is used, its shape functions have Kronecker-delta property. Therefore, the unsatisfactory issue of the essential node condition can be avoided in the modified MLS approximation.

Consider a small domain $\Omega_{x}$, the neighborhood of a point $x_{1}$, which is located in $\Omega$ or on $\Gamma$. Over a number of randomly located nodes $\left\{x_{i}\right\}, i=1,2, \cdots, n$, the MLS approximation $u^{h}$ of $u$ can be defined by

$$
u^{h}=\mathbf{p}^{T}(\mathbf{x}) \boldsymbol{\alpha}, \quad \forall \mathbf{x} \in \Omega_{x}
$$

where $\mathbf{p}^{T}(\mathbf{x})=\left[\begin{array}{llll}p_{1}(\mathbf{x}) & p_{2}(\mathbf{x}) & \cdots & p_{m}(\mathbf{x})\end{array}\right]$ is a complete monomial basis of order $\mathrm{m}$ which is a function of the space coordinates $\mathbf{x}=\left[\begin{array}{lll}x & y & z\end{array}\right]^{T} . \boldsymbol{\alpha}$ is a vector of unknown polynomial coefficients.

$$
\boldsymbol{\alpha}=\left[\begin{array}{llll}
\alpha_{1} & \alpha_{2} & \cdots & \alpha_{m}
\end{array}\right]^{T}
$$

For example, for a 2-D problem,

$$
\mathbf{p}^{T}(\mathbf{x})=\left[\begin{array}{llllll}
1 & x & y & x^{2} & x y & y^{2}
\end{array}\right]
$$

this is a quadratic basis, and $m=6$.

A weighted least-square solution is obtained for $\boldsymbol{\alpha}$ from the following system of $n$ equations in $m$ unknown ( $n$ is larger than $m$ ):

$$
\mathbf{u}^{h}=\mathbf{H} \boldsymbol{\alpha}
$$

where

$$
\mathbf{u}^{h}=\left[\begin{array}{llll}
u_{1}^{h} & u_{2}^{h} & \cdots & u_{n}^{h}
\end{array}\right]^{T}
$$

is a vector of the nodal MLS approximation of function $u$, and

$$
\mathbf{H}=\left[\begin{array}{c}
\mathbf{p}^{T}\left(\mathbf{x}_{1}\right) \\
\mathbf{p}^{T}\left(\mathbf{x}_{2}\right) \\
\vdots \\
\mathbf{p}^{T}\left(\mathbf{x}_{n}\right)
\end{array}\right]_{n \times m}
$$

The classical least-square solution of the above over-constrained system does not guarantee exact satisfaction of any of the equations of Eq. (7). Non-satisfaction of the first equation would then mean $u_{1}^{h} \neq p^{T}\left(x_{1}\right) \alpha$. Hence, a different approach to weighted least-squares 
solution can be adopted: Out of the $n$ equations of Eq. (7), let the first equation (corresponding to node 1) be satisfied exactly and the rest in the least-square sense. This is done by using the first equation to eliminate $\alpha_{1}$ from the rest of equations:

$$
\alpha_{1}=u_{1}^{n}-\left(\alpha_{2} x_{1}+\alpha_{3} y_{1}+\alpha_{3} x_{1}^{2}+\alpha_{5} x_{1} y_{1}+\alpha_{6} y_{1}^{2}\right)
$$

Substituting for $\alpha_{1}$ in Eq. (7), the reduced system of equations can be obtained:

$$
\overline{\mathbf{u}}^{h}=\overline{\mathbf{H}} \overline{\boldsymbol{\alpha}}
$$

where

$$
\begin{aligned}
& \overline{\mathbf{u}}^{h}=\left[\begin{array}{llll}
u_{2}^{h}-u_{1}^{h} & u_{3}^{h}-u_{1}^{h} & \cdots & u_{n}^{h}-u_{1}^{h}
\end{array}\right]^{T} \\
& \overline{\mathbf{H}}=\left[\begin{array}{ccccc}
x_{2}-x_{1} & y_{2}-y_{1} & x_{2}^{2}-x_{1}^{2} & x_{2} y_{2}-x_{1} y_{1} & y_{2}^{2}-y_{1}^{2} \\
x_{3}-x_{1} & y_{3}-y_{1} & x_{3}^{2}-x_{1}^{2} & x_{3} y_{3}-x_{1} y_{1} & y_{3}^{2}-y_{1}^{2} \\
\vdots & \vdots & \vdots & \vdots & \vdots \\
x_{n}-x_{1} & y_{n}-y_{1} & x_{n}^{2}-x_{1}^{2} & x_{n} y_{n}-x_{1} y_{1} & y_{n}^{2}-y_{1}^{2}
\end{array}\right]=\left[\begin{array}{c}
\overline{\mathbf{p}}^{T}\left(\mathbf{x}_{2}\right) \\
\overline{\mathbf{p}}^{T}\left(\mathbf{x}_{3}\right) \\
\vdots \\
\overline{\mathbf{p}}^{T}\left(\mathbf{x}_{n}\right)
\end{array}\right] \\
& \overline{\boldsymbol{\alpha}}=\left[\begin{array}{llll}
\alpha_{2} & \alpha_{3} & \cdots & \alpha_{m}
\end{array}\right]^{T}
\end{aligned}
$$

The coefficient vector $\overline{\boldsymbol{\alpha}}$ is determined by minimizing a weighted discrete $L_{2}$ norm, defined as:

$$
J=\sum_{i=2}^{n} w\left(\mathbf{x}_{i}\right)\left[\overline{\mathbf{p}}^{T}\left(\mathbf{x}_{i}\right) \bar{\alpha}-\bar{u}_{i}\right]^{2}=[\overline{\mathbf{H}} \overline{\boldsymbol{\alpha}}-\overline{\mathbf{u}}]^{T} \mathbf{W}[\overline{\mathbf{H}} \overline{\boldsymbol{\alpha}}-\overline{\mathbf{u}}]
$$

where $w(\mathbf{x})$ is the weight function, with $w(\mathbf{x})>0$ for all nodes in the support of $w(\mathbf{x})$ (the support is considered to be equal to $\Omega_{x}$ in this paper), $\mathbf{x}_{i}$ denotes the value of $\mathbf{x}$ at node $i$, and the matrices $\mathbf{W}$ is defined as

$$
\begin{aligned}
& \mathbf{W}=\left[\begin{array}{cccc}
w\left(\mathbf{x}_{2}\right) & 0 & \cdots & 0 \\
0 & w\left(\mathbf{x}_{3}\right) & \cdots & 0 \\
\cdots & \cdots & \cdots & \cdots \\
0 & 0 & \cdots & w\left(\mathbf{x}_{n}\right)
\end{array}\right]_{(n-1) \times(n-1)} \\
& \bar{u}_{i}=\hat{u}_{i}-\hat{u}_{1}, \quad i=2,3, \cdots, n \\
& \overline{\mathbf{u}}=\left[\begin{array}{llll}
\hat{u}_{2}-\hat{u}_{1} & \hat{u}_{3}-\hat{u}_{1} & \cdots & \hat{u}_{n}-\hat{u}_{1}
\end{array}\right]^{T}
\end{aligned}
$$

where $\hat{u}_{i}, i=1,2, \cdots, n$, are the fictitious nodal values of the function $u$.

Minimizing $J$ in Eq. (15) with respect to $\overline{\boldsymbol{\alpha}}$ yields

$$
\begin{aligned}
& \overline{\boldsymbol{\alpha}}=\mathbf{A}^{-1} \mathbf{B} \overline{\mathbf{u}} \\
& \mathbf{B}=\overline{\mathbf{H}}^{T} \mathbf{W} \\
& \mathbf{A}=\mathbf{B} \overline{\mathbf{H}}
\end{aligned}
$$

Substituting Eq. (19) into Eq. (11) gives a relation which may be written as the form of an interpolation function, as

$$
\overline{\mathbf{u}}^{h}=\overline{\mathbf{H}} \mathbf{A}^{-1} \mathbf{B} \overline{\mathbf{u}}
$$

Eq. (10) can be rewritten as:

$$
\begin{aligned}
& \alpha_{1}=u_{1}^{h}-\mathbf{s}\left(\mathbf{x}_{1}\right) \bar{\alpha} \\
& \mathbf{s}\left(\mathbf{x}_{1}\right)=\left[\begin{array}{lllll}
x_{1} & y_{1} & x_{1}^{2} & x_{1} y_{1} & y_{1}^{2}
\end{array}\right]
\end{aligned}
$$


Equation (4) can be written as:

$$
\begin{aligned}
& u^{h}=\alpha_{1}+\mathbf{s}(\mathbf{x}) \bar{\alpha} \\
& \mathbf{s}(\mathbf{x})=\left[\begin{array}{lllll}
x & y & x^{2} & x y & y^{2}
\end{array}\right]
\end{aligned}
$$

Substituting Eq. (19) and Eq. (23) into Eq. (25), the following equation can be obtained:

$$
\begin{aligned}
& u^{h}=u_{1}^{h}+\mathbf{q}(\mathbf{x}) \mathbf{A}^{-1} \mathbf{B} \overline{\mathbf{u}} \\
& \mathbf{q}(\mathbf{x})=\mathbf{s}(\mathbf{x})-\mathbf{s}\left(\mathbf{x}_{1}\right)
\end{aligned}
$$

Because

$$
\begin{aligned}
& \mathbf{q}\left(\mathbf{x}_{1}\right)=0 \\
& u^{h}\left(\mathbf{x}_{1}\right)=u_{1}^{h}
\end{aligned}
$$

$\widehat{\mathbf{u}}$ may be defined as

$$
\widehat{\mathbf{u}}=\left[\begin{array}{llll}
\hat{u}_{1} & \hat{u}_{2} & \cdots & \hat{u}_{n}
\end{array}\right]^{T}
$$

then, from Eq. (27), the following equation may be obtained:

$$
\begin{aligned}
& u^{h}=\mathbf{N}(\mathbf{x}) \widehat{\mathbf{u}} \\
& \underset{1 \times n}{\mathbf{N}(\mathbf{x})}=\left[1-\left(\underset{1 \times(\mathrm{m}-1)}{\mathbf{q}(\mathbf{x})} \underset{(\mathrm{m}-1) \times(m-1)}{\mathbf{A}^{-1}} \underset{(\mathrm{m}-1) \times(n-1)}{\mathbf{B}} \underset{(\mathrm{n}-1) \times 1}{\mathbf{1}}\right) \vdots \underset{1 \times(\mathrm{m}-1)}{\mathbf{q}(\mathbf{x})} \underset{(\mathrm{m}-1) \times(m-1)}{\mathbf{\mathbf { A } ^ { - 1 }}} \underset{(\mathrm{m}-1) \times(n-1)}{\mathbf{B}}\right]
\end{aligned}
$$

In Eq. (33), 1 is vector of dimension ( $n-1)$ with all entries being equal to unity.

Recall from Eq. (29), using this result in Eq. (33), the Kronecker-delta property of $\mathbf{N}(\mathbf{x})$ may be established:

$$
\mathbf{N}\left(\mathbf{x}_{1}\right)=\left[\begin{array}{lllll}
1 & 0 & 0 & \cdots & 0
\end{array}\right]
$$

It means that at node 1 , the shape function for node 1 takes a value of unity and all other shape function take zero values. Therefore, Eq. (33) is the shape functions of the MLS approximation with Kronecker-delta property.

From Eq. (32) and Eq. (30), the following result can be obtained:

$$
\hat{u}_{1}=u^{h}\left(\mathbf{x}_{1}\right)=u_{1}^{h}
$$

In this paper, the weight functions $w(\mathbf{x})$ may use a spline function as follows:

$$
\begin{aligned}
& w(\mathbf{x})=1-6\left(\frac{d}{r}\right)^{2}+8\left(\frac{d}{r}\right)^{3}-3\left(\frac{d}{r}\right)^{4}, \quad 0 \leq d \leq r \\
& w(\mathbf{x})=0, \quad d \geq r
\end{aligned}
$$

where $d=\left|\mathbf{x}-\mathbf{x}_{1}\right|$ is the distance from point $x$ to the center node $x_{1}$, and $r$ is the radius of $\Omega_{x}$, which is taken as a circle for a 2-D problem and its center is the point $x_{1}$.

As the MLS approximation $u^{h}$ of $u$, Equation (32) can be substituted into Eqs. (1), (2) and (3). Therefore, the algebraic equations on $\hat{u}_{i},\left(i=1,2, \cdots, N_{f}\right.$, where $N_{f}$ is the total number of freedoms over $\Omega$ ) can be obtained, and the number of the equations can be selected as $N_{f}$ by using the collocation scheme, too. 


\subsection{The Positivity Conditions}

The positivity conditions ${ }^{(4)}$ on the approximation function $N_{i}(\mathbf{x})$ of Eq. (33) and its second-order derivatives are stated as,

$$
\begin{aligned}
& N_{i}\left(\mathbf{x}_{j}\right) \geq 0 \\
& \nabla^{2} N_{i}\left(\mathbf{x}_{j}\right) \geq 0, j \neq i \\
& \nabla^{2} N_{i}\left(\mathbf{x}_{i}\right)<0
\end{aligned}
$$

where $N_{i}\left(\mathbf{x}_{j}\right)$ is the approximation function of a point $i$ evaluated at a point $j$.

Patankar ${ }^{(12)}$ included the positivity conditions in a series of basic rules for the construction of finite differences and pointed out that the consequence of violating the positivity conditions give a physically unrealistic solution. It has been shown that the satisfaction of the positivity conditions ensures the convergence of the finite difference method with arbitrary irregular meshes for some class of elliptic problems ${ }^{(13)}$. It has been shown that the significance of the positivity conditions in meshless collocation approaches, and violation of the positivity conditions can significantly result in a large error in the numerical solution ${ }^{(4)}$.

For a point $\boldsymbol{x}_{1}$ on $\Gamma$, if no over-range point is used in its $\Omega_{x}$, the positivity conditions on the boundary point cannot be satisfied, obviously. But by introducing some over-range points of $\Omega$ in the $\Omega_{x}$, the unsatisfactory issue of the positivity conditions of the boundary point can be avoided in the ORCM

\section{Numerical Implementation}

\subsection{Method of Error Estimation}

For the purpose of error estimation and convergence studies, the Sobolev norm $\|u\|_{0}$, of function $u$ and the norm of the first-order derivative vector of $u,\|\mathbf{q}\|_{0}$ are calculated. These norms are defined as

$$
\begin{aligned}
& \|u\|_{0}=\left(\int_{\Omega} u^{2} d \Omega\right)^{1 / 2} \\
& \|\mathbf{q}\|_{0}=\left(\int_{\Omega} \mathbf{q}^{T} \cdot \mathbf{q} d \Omega\right)^{1 / 2} \\
& \mathbf{q}=\left[\begin{array}{ll}
\partial u / \partial x & \partial u / \partial y
\end{array}\right]^{T}=\left[\begin{array}{ll}
q_{x} & q_{y}
\end{array}\right]^{T}
\end{aligned}
$$

The relative errors for $\|u\|_{0}$ and $\|\mathbf{q}\|_{0}$ are defined as

$$
\begin{aligned}
& R_{0}=\frac{\left\|u^{n u m}-u^{\text {exa }}\right\|_{0}}{\left\|u^{\text {exa }}\right\|_{0}} \\
& R_{q}=\frac{\left\|\mathbf{q}^{\text {num }}-\mathbf{q}^{\text {exa }}\right\|_{0}}{\left\|\mathbf{q}^{\text {exa }}\right\|_{0}}
\end{aligned}
$$

\subsection{Poisson's Boundary Value Problems}

Some boundary value problems have been analyzed by using the ORCM, and it has been shown that the ORCM works well for those boundary value problems ${ }^{(11),(14)}$.

In this paper, a Poisson's equation with a quadratic source function

$$
\nabla^{2} \mathrm{u}=10\left(x^{2}+y^{2}\right)
$$

is analyzed over the $10 \times 10$ domain ( see Fig. 2 ) by using the ORCM, and its numerical solutions are compared with the exact solutions:

$$
\mathrm{u}=x^{4}+y^{4}-x^{3} y-x^{2} y^{2}+x y^{3}
$$


A Dirichlet problem, for which the essential boundary condition $\left(u=u_{c}\right)$ is imposed at nodes on all boundaries, and two mixed problem, the first mixed problem ( the essential boundary condition is imposed at nodes on left and right boundaries and the flux boundary condition ( $\left.q_{y}=t_{y}\right)$ is prescribed at nodes on top and bottom boundaries ) and the second mixed problem ( the essential boundary condition is imposed at nodes on top and bottom boundaries and the flux boundary condition $\left(q_{x}=t_{\mathrm{x}}\right)$ is prescribed at nodes on left and right boundaries ), are solved, where $t_{\mathrm{x}}$ and $t_{\mathrm{y}}$ are the assigned values of $q_{x}$ and $q_{y}$ over $\Gamma_{t}$, respectively. To meet the condition of $K_{o}=K_{b}, 8$ nodes are added to the boundary points, which are located on the boundary near the four corners of the domain, for all nodal models in this paper. Regular nodal models (including the over-range nodes) of $177(13 \times 13+8)\left(K_{d}=9 \times 9, K_{b}=K_{o}=48\right)$ nodes, $233(15 \times 15+8)\left(K_{d}=11 \times 11, K_{b}=K_{o}=56\right)$

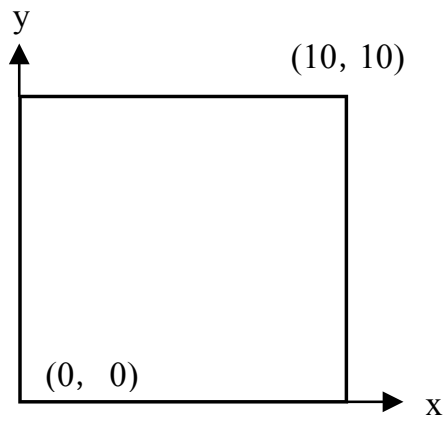

Fig. 2 Analyzed domain

nodes and $297(17 \times 17+8)\left(K_{d}=13 \times 13, K_{b}=K_{o}=64\right)$ nodes are used to study the convergence with nodal model refinement of the ORCM. Over-range points of one layer are used, and the over-range points are regularly located at outside of the four sides of the domain. Figure 3 (a) shows nodal distribution of $233(15 \times 15+8)\left(K_{d}=11 \times 11, K_{b}=K_{o}=56\right)$ regular nodal model.

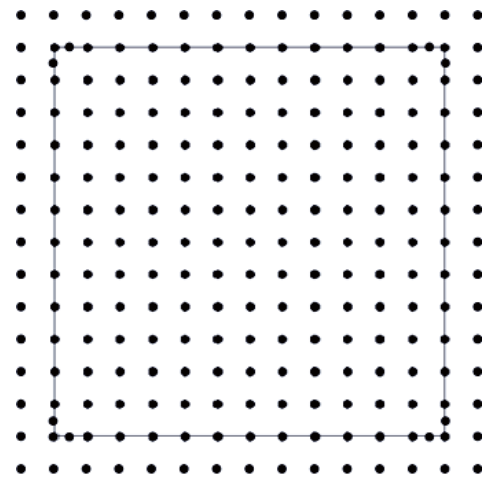

(a) Regular nodal model

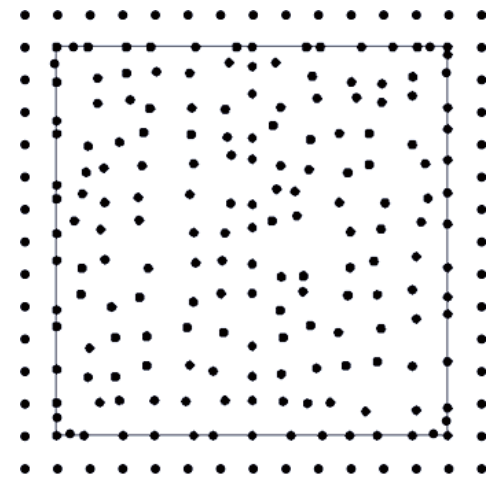

(b) Irregular nodal model

Fig. 3 Nodal distribution of 233 node models

To discuss the convergences with nodal model refinement of the ORCM, the convergence rates $c_{i}$ are calculated, which are defined as:

$$
c_{i+1}=\frac{\log _{10}\left(R_{i} / R_{i+1}\right)}{\log _{10}\left(h_{i} / h_{i+1}\right)}
$$

where $R=R_{0}, R_{q}, h$ is the nodal spacing, and the subscripts " $i$ " and " $i+l$ " denote two consecutive uniform $h$-refinements. 
The results of relative errors and convergence rates are shown in Fig. 4, Fig. 5 and Fig. 6 for the Dirichlet problem, the first mixed problem, and the second mixed problem, respectively. In these figures, $c$ is the average convergence rate of the three consecutive uniform $h$-refinements, and the error convergences can be observed for the three boundary value problems. Additionally, the error estimation norms of weak form ( see Eqs. (40) and (41) ) are used for the strong form method ( collocation method ) in this paper. It is a severe error estimation method, nevertheless, the order of the relative error is about $10^{-2}$ or $10^{-3}$ in Figs. 4, 5 and 6. It is shown that the ORCM works well for the three boundary value problems.

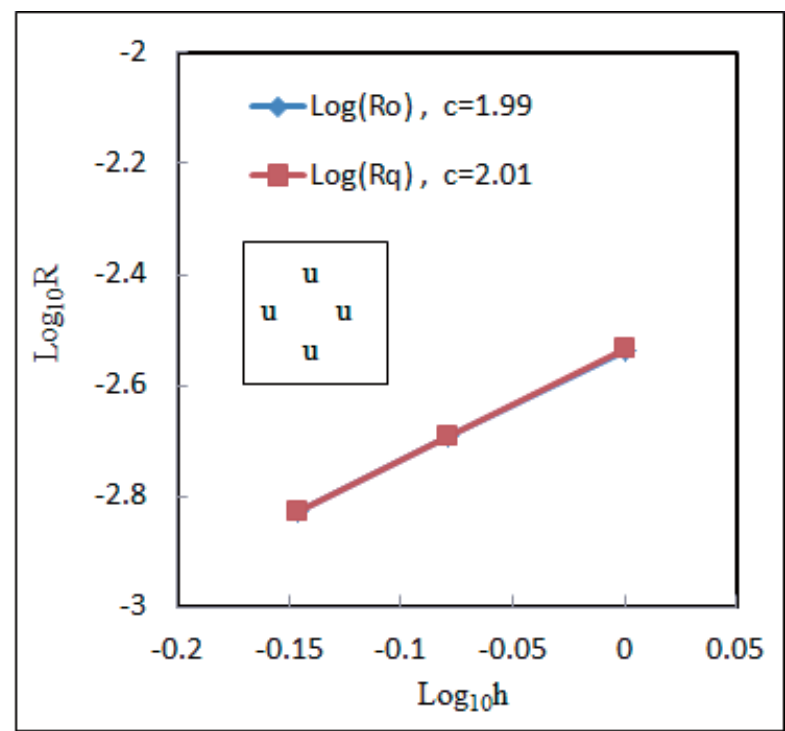

Fig. 4 Relative errors and convergences for the Dirichlet problem

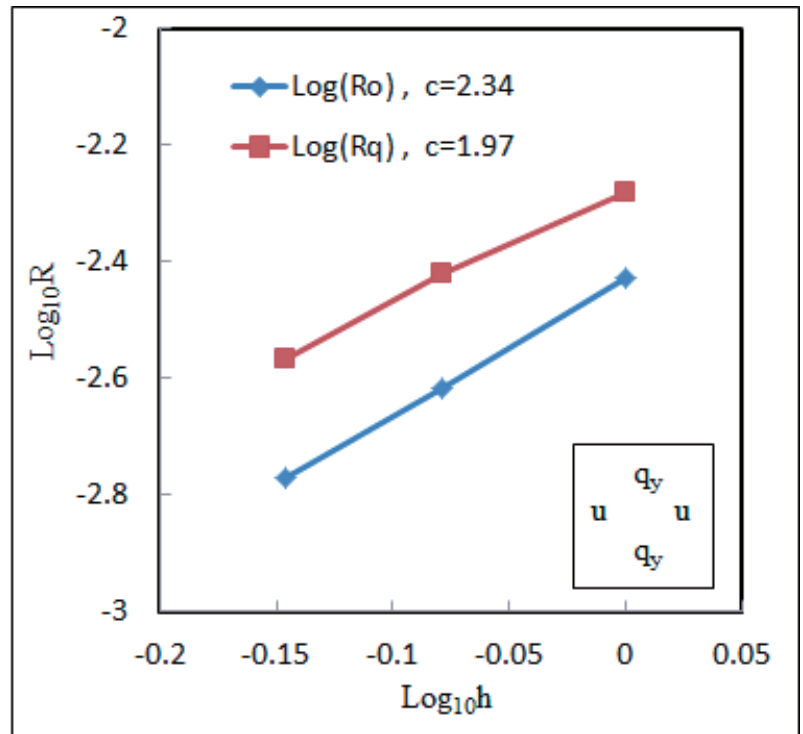

Fig. 5 Relative errors and convergences for the first mixed problem 


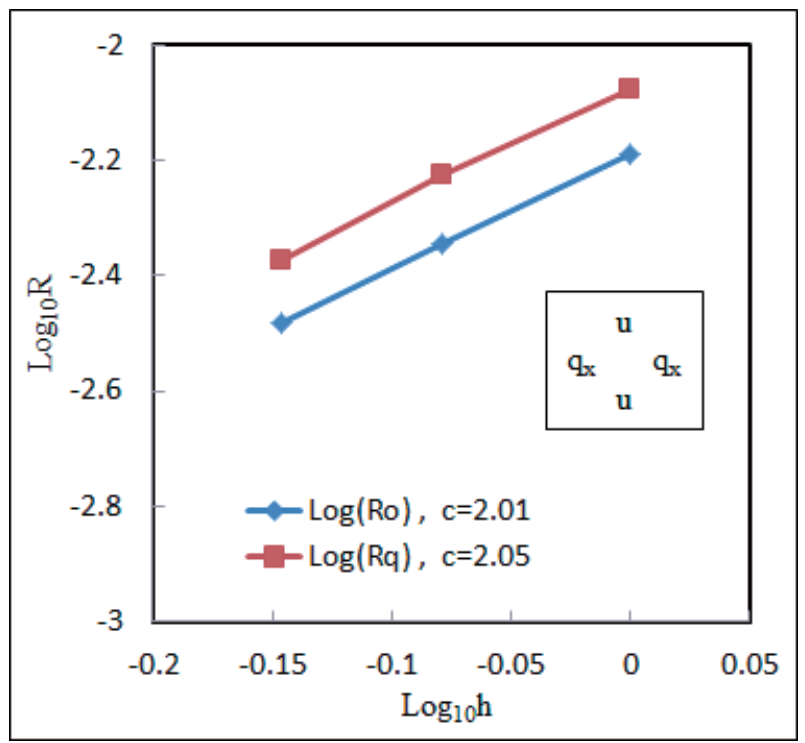

Fig. 6 Relative errors and convergences for the second mixed problem

Figures 7, 8 and 9 show values of $\mathrm{u}$ at $x=5.0$ by regular nodal model of 233 nodes, for Dirichlet problem, respectively. Figures 10,11 and 12 show values of $u$ at $x=5.0$ by regular nodal model of 233 nodes, for the first mixed problem, respectively. Figures 13, 14 and 15 show values of $u$ at $x=5.0$ by regular nodal model of 233 nodes, for the second mixed problem, respectively. It can be seen that some accurate results of the unknown variable and its derivatives for the three boundary value problems are obtained by using the regular nodal model.

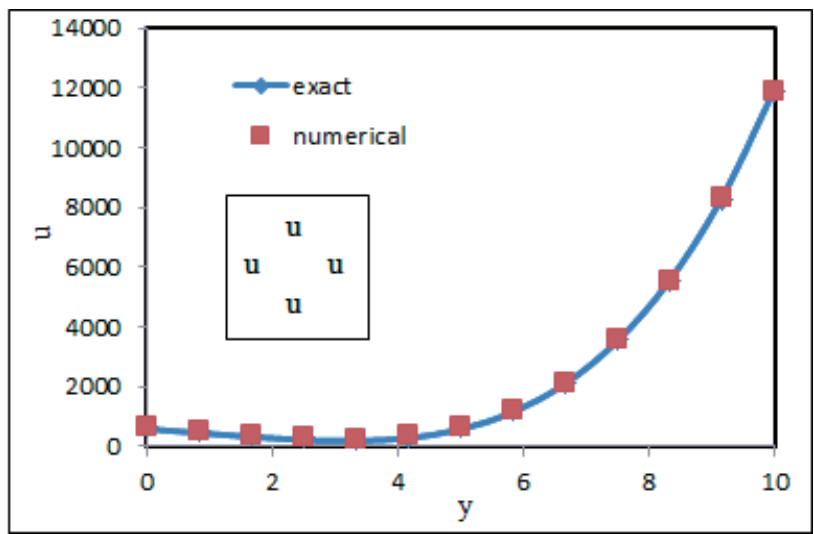

Fig. 7 Values of $u$ at $x=5.0$ by regular nodal model of 233 nodes, for the Dirichlet problem 


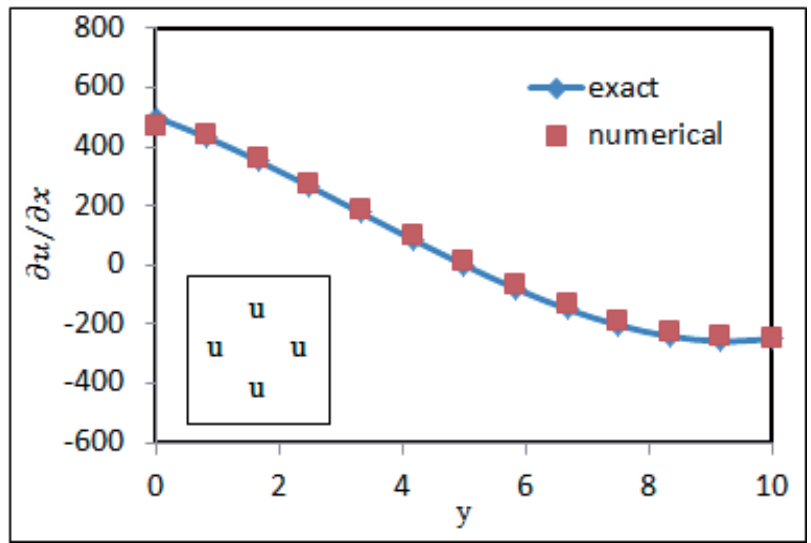

Fig. 8 Values of $\partial \mathrm{u} / \partial \mathrm{x}$ at $x=5.0$ by regular nodal model of 233 nodes, for the Dirichlet problem

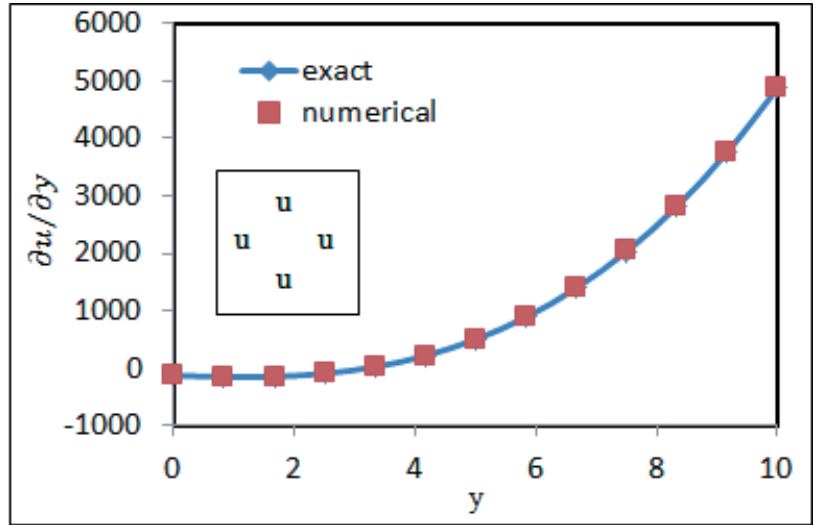

Fig. 9 Values of $\partial \mathrm{u} / \partial \mathrm{y}$ at $x=5.0$ by regular nodal model of 233 nodes, for the Dirichlet problem

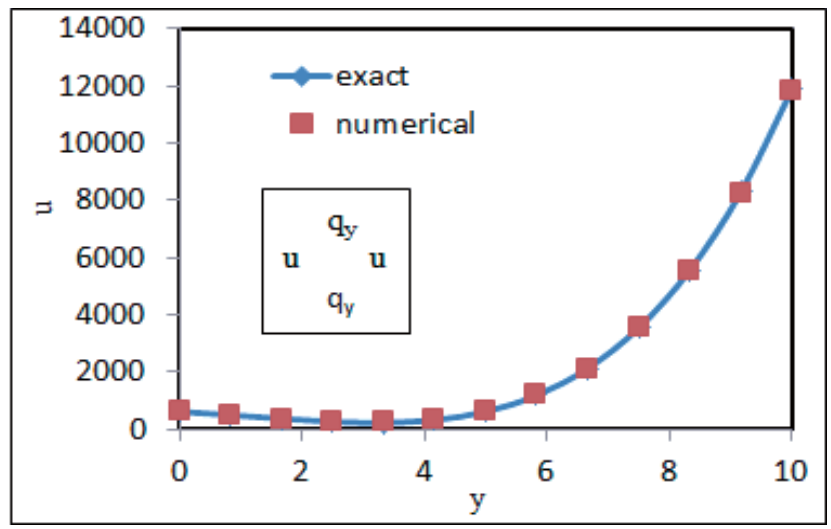

Fig. 10 Values of $u$ at $x=5.0$ by regular nodal model of 233 nodes, for the first mixed problem 


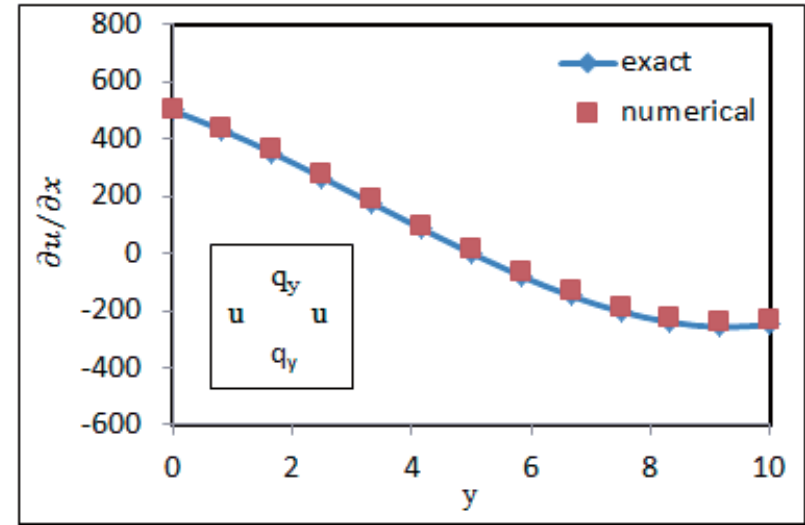

Fig. 11 Values of $\partial \mathrm{u} / \partial \mathrm{x}$ at $x=5.0$ by regular nodal model of 233 nodes, for the first mixed problem

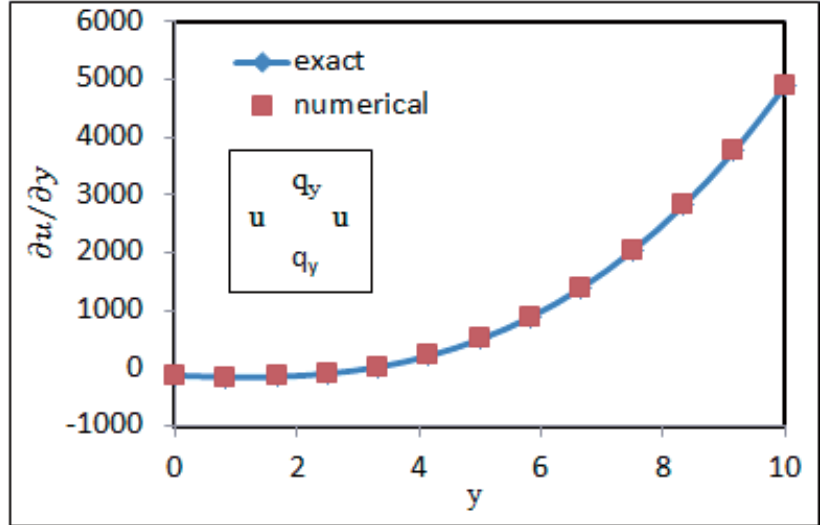

Fig. 12 Values of $\partial \mathrm{u} / \partial \mathrm{y}$ at $x=5.0$ by regular nodal model of 233 nodes, for the first mixed problem

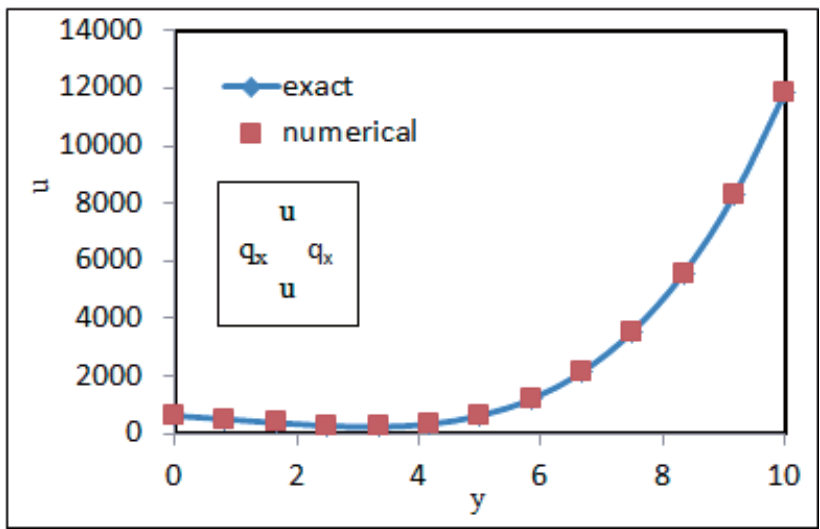

Fig. 13 Values of $u$ at $x=5.0$ by regular nodal model of 233 nodes, for the second mixed problem 


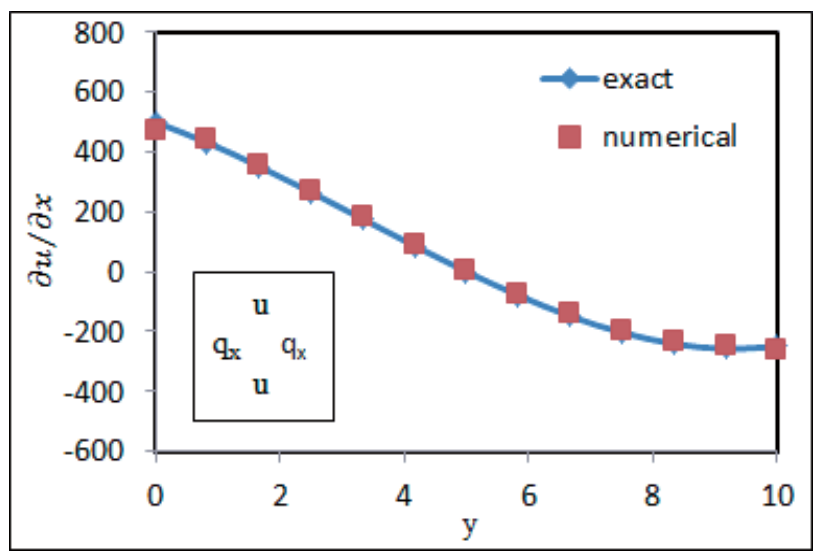

Fig. 14 Values of $\partial \mathrm{u} / \partial \mathrm{x}$ at $x=5.0$ by regular nodal model of 233 nodes, for the second mixed problem

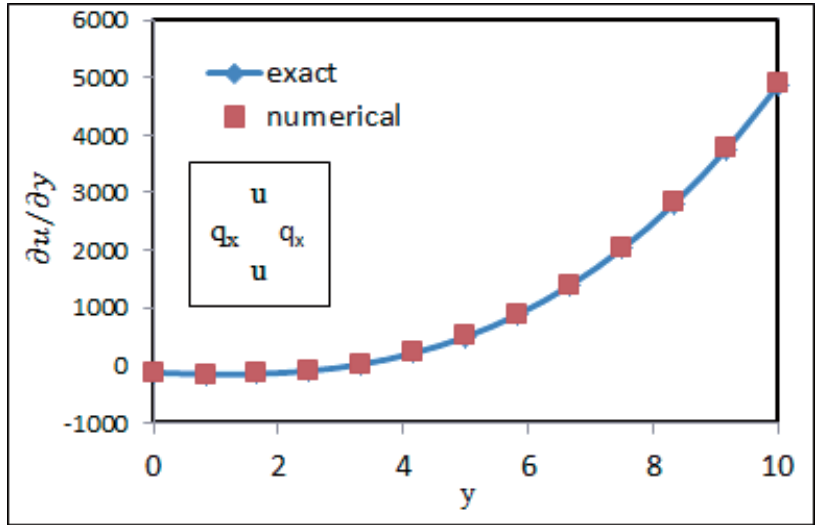

Fig. 15 Values of $\partial \mathrm{u} / \partial \mathrm{y}$ at $x=5.0$ by regular nodal model of 233 nodes, for the second mixed problem

One irregular nodal model of $233\left(K_{d}=121, K_{b}=K_{o}=56\right)$ nodes is used, too. Figure 3(b) shows nodal distribution of $233\left(K_{d}=121, K_{b}=K_{o}=56\right)$ irregular nodal model. Figures 16 , 17 and 18 show values of $u$ at $x=5.0$ by irregular nodal model of 233 nodes, for Dirichlet problem, respectively. Figures 19, 20 and 21 show values of $u$ at $x=5.0$ by irregular nodal model of 233 nodes, for the first mixed problem, respectively. Figures 22, 23 and 24 show values of $u$ at $x=5.0$ by irregular nodal model of 233 nodes, for the second mixed problem, respectively. It can be seen that some accurate results of the unknown variable and its derivatives for the three boundary value problems are obtained by using the irregular nodal model, too (excepting value of $\partial \mathrm{u} / \partial \mathrm{x}$ of center point at the bottom side $(x=5.0, y=0.0)$ for the first mixed problem by using the irregular nodal model ( see Fig. 20 ) ). 


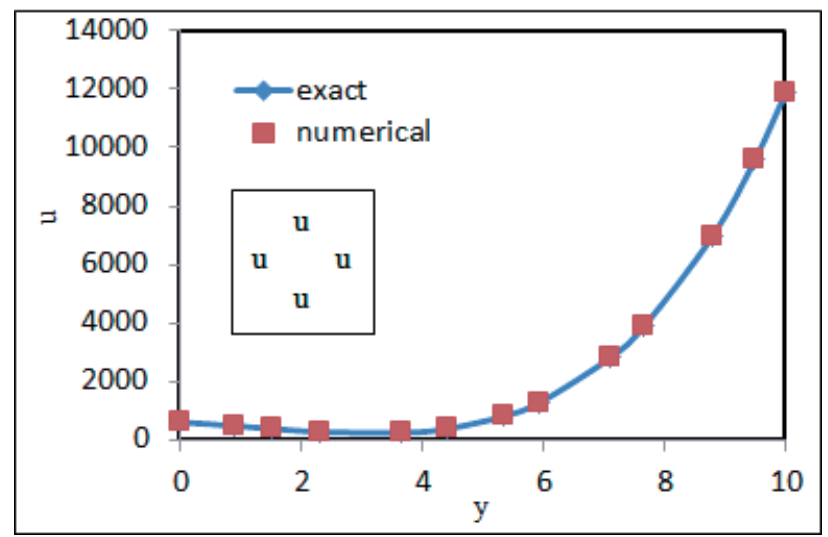

Fig. 16 Values of $u$ at $x=5.0$ by irregular nodal model of 233 nodes, for the Dirichlet problem

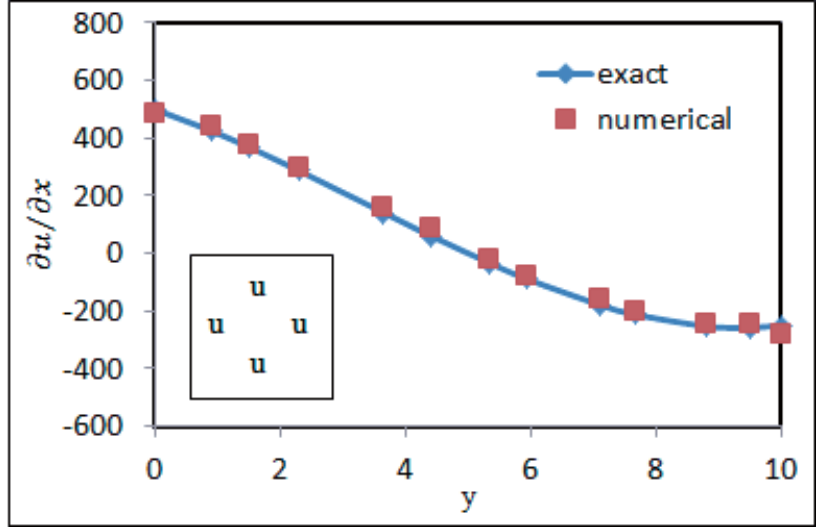

Fig. 17 Values of $\partial \mathrm{u} / \partial \mathrm{x}$ at $x=5.0$ by irregular nodal model of 233 nodes, for the Dirichlet problem

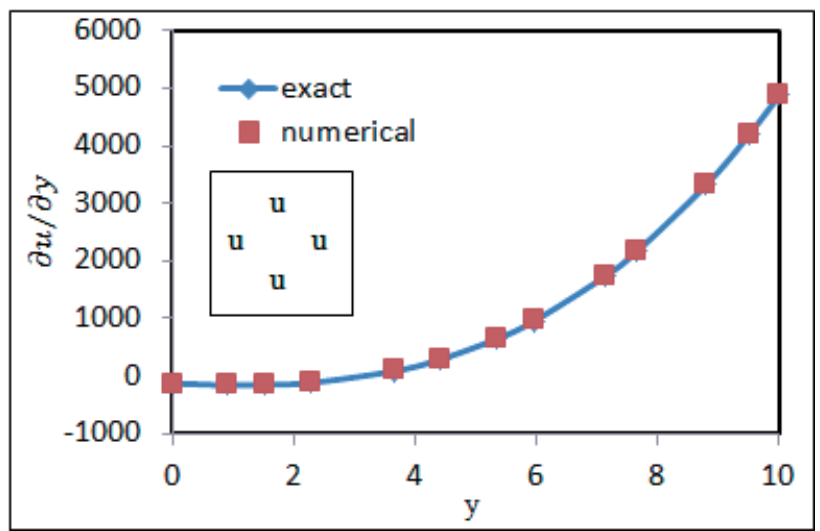

Fig. 18 Values of $\partial \mathrm{u} / \partial \mathrm{y}$ at $x=5.0$ by irregular nodal model of 233 nodes, for the Dirichlet problem 


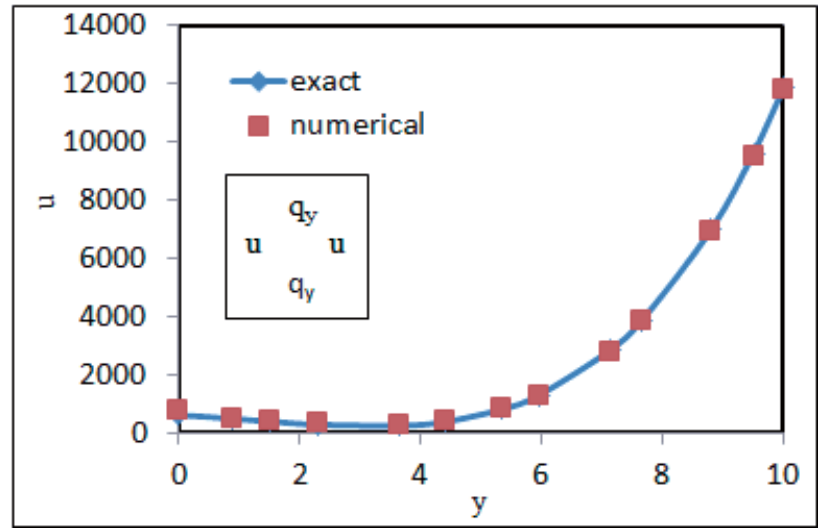

Fig. 19 Values of $u$ at $x=5.0$ by irregular nodal model of 233 nodes, for the first mixed problem

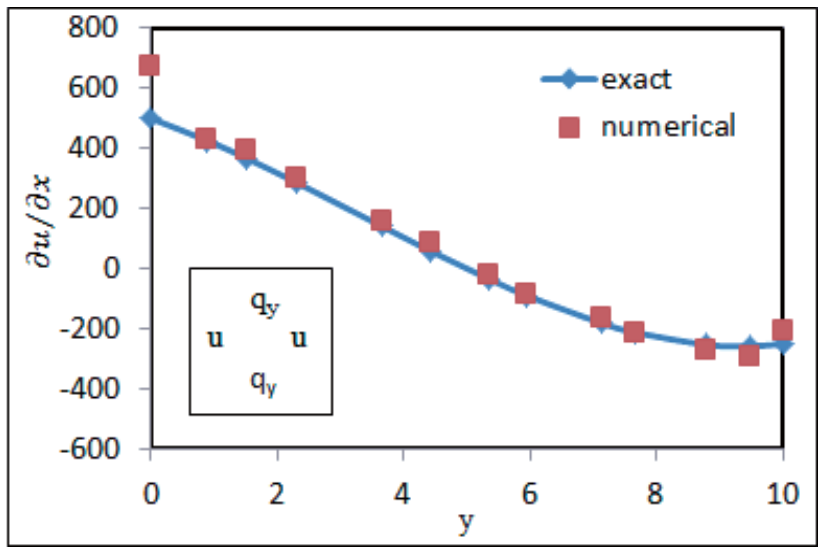

Fig. 20 Values of $\partial \mathrm{u} / \partial \mathrm{x}$ at $x=5.0$ by irregular nodal model of 233 nodes, for the first mixed problem

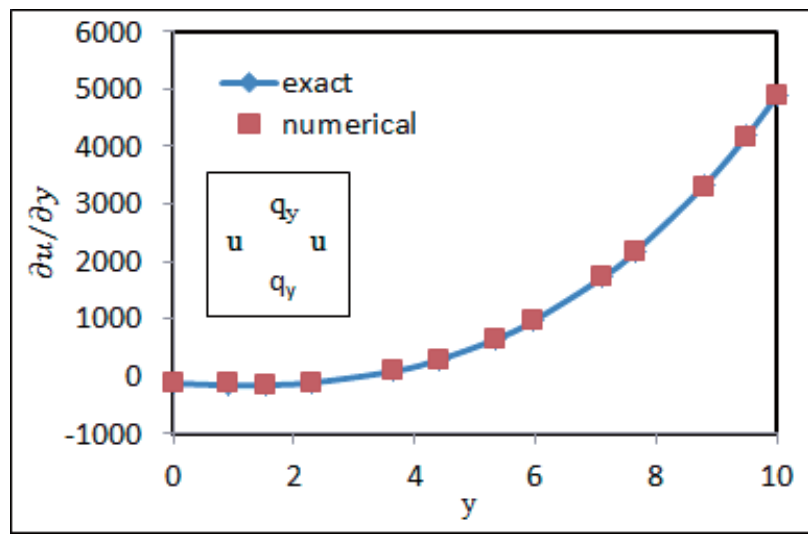

Fig. 21 Values of $\partial \mathrm{u} / \partial \mathrm{y}$ at $x=5.0$ by irregular nodal model of 233 nodes, for the first mixed problem 


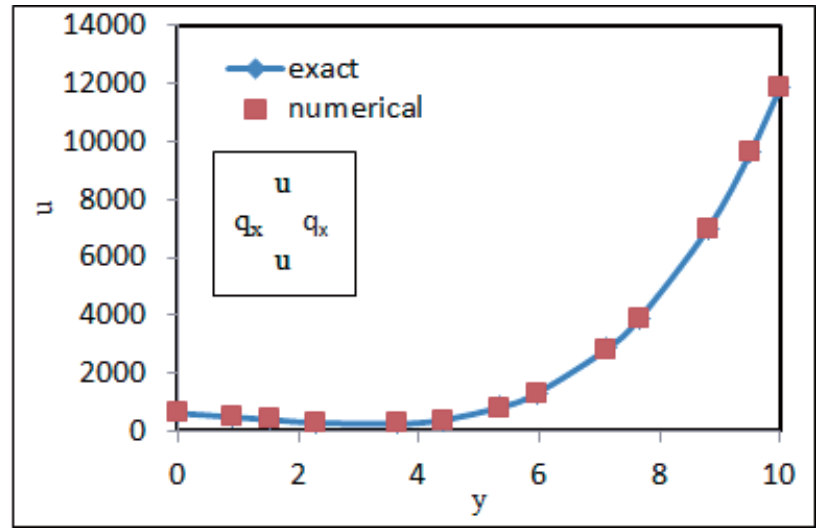

Fig. 22 Values of $u$ at $x=5.0$ by irregular nodal model of 233 nodes, for the second mixed problem

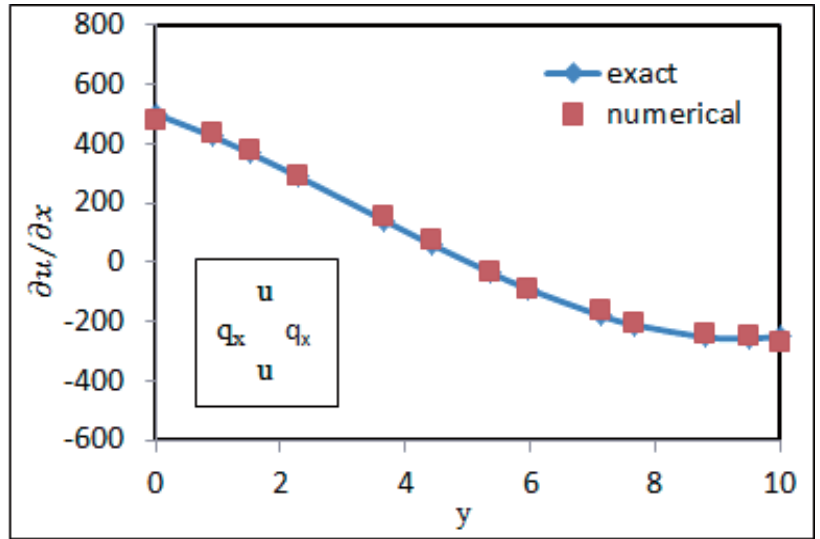

Fig. 23 Values of $\partial \mathrm{u} / \partial \mathrm{x}$ at $x=5.0$ by irregular nodal model of 233 nodes, for the second mixed problem

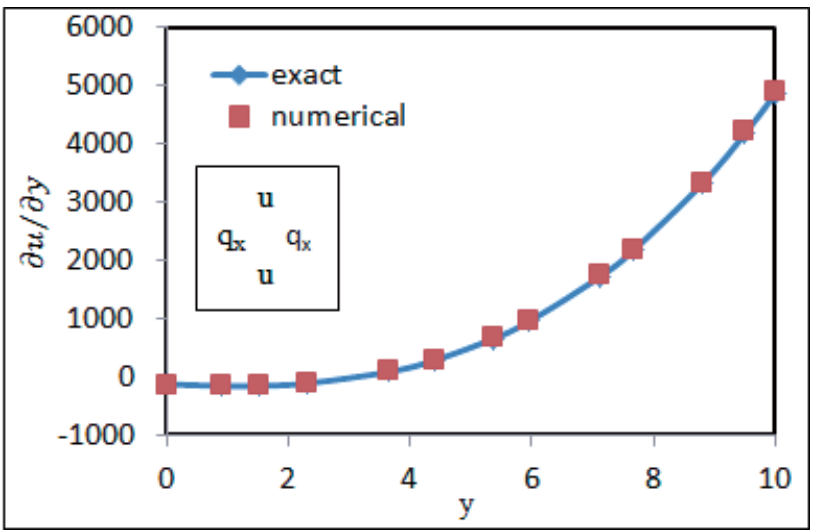

Fig. 24 Values of $\partial \mathrm{u} / \partial \mathrm{y}$ at $x=5.0$ by irregular nodal model of 233 nodes, for the second mixed problem

\section{Conclusions}

The three boundary value problems on Poisson's equation are analyzed by using the ORCM, in which by introducing some collocation points that are located at outside of domain of the analyzed body, unsatisfactory issue of the positivity conditions of boundary points in collocation methods can be avoided. The convergence studies show that the ORCM possesses good convergence for both the unknown variables and their derivatives. Quite accurate numerical results calculated by both regular nodal models and irregular nodal models have been obtained. The ORCM does not demand any specific kind of partial 
differential equations, therefore it shows promise of wide engineering applications of the ORCM.

\section{References}

(1) Onate, E., Idelsohn, S., Zienkiewicz, O.C. and Toylor, R.L., A Finite Point Method in Computational Mechanics, Applications to Convective Transport and Fluid Flow, International Journal for Numerical Methods in Engineering, Vol. 39 (1996), pp. 3839-3866.

(2) Onate, E., Idelsohn, S., Zienkiewicz, O.C., Toylor, R.L. and Sacco, C., A Stabilized Finite Point Method for Analysis of Fluid Mechanics Problems, Computer Methods in Applied Mechanics and Engineering, Vol. 139 (1996), pp. 315-346.

(3) Aluru, N.R., A Point Collocation Method Based on Reproducing Kernel Approximations, International Journal for Numerical Methods in Engineering, Vol. 47 (2000), pp. 1083-1121.

(4) Jin, X., Li, G. and Aluru, N.R., Positivity Conditions in Meshless Collocation Methods, Computer Methods in Applied Mechanics and Engineering, Vol. 193 (2004), pp. 1171-1202.

(5) Boroomand, B., Tabatabaei, A.A. and Onate, E., Simple Modifications for Stabilization of the Finite Point Method. International Journal for Numerical Methods in Engineering, Vol. 63 (2005), pp. 351-379.

(6) Atluri, S.N., Liu, H.T. and Han, Z.D., Meshless Local Petrov-Galerkin (MPLG) Mixed Collocation Method for Elasticity Problems, Computer Modeling in Engineering \& Sciences, Vol. 14 (2006), pp. 141-152.

(7) Atluri, S. N., Liu, H.T. and Han, Z.D., Meshless local Petrov-Galerkin (MPLG) Mixed Finite Difference Method for Solid Mechanics. Computer Modeling in Engineering \& Sciences, Vol. 15 (2006), pp. 1-16.

(8) Li, Shu and Atluri, S.N., The MPLG Mixed Collocation Method for Material Orientation and Topology Optimization of Anisotropic Solids and Structures. Computer Modeling in Engineering \& Sciences, Vol. 30 (2008), pp. 37-56.

(9) Kosec, G. and Sarler, B., Local RBF Collocation Method for Darcy Flow, Computer Modeling in Engineering \& Sciences, Vol. 25 (2008), pp. 197-207.

(10) Wu, C.-P., Chiu, K.-H. and Wang, Y.-M., A Mesh-Free DRK-Based Collocation Method for the Coupled Analysis of Functionally Graded Magneto-Electro-Elastic Shells and Plates, Computer Modeling in Engineering \& Sciences, Vol. 35 (2008), pp. 181-214.

(11) Guo, Y.-M., An Overrange Collocation Method, Computer Modeling in Engineering \& Sciences, Vol. 73 (2011), pp .1-22.

(12) Patanakar, S.V., Numerical Heat Transfer and Fluid Flow, Hemisphere, (1980).

(13) Demkowicz, L., Karafilt, A. and Liszka, T., On Some Convergence Results for FDM with Irregular Mesh, Computer Methods in Applied Mechanics and Engineering, Vol. 42 (1984), pp. 343-355.

(14) Guo, Y.-M., Osako, H. and Kamitani, S., Nonlinear Analyses by Using the ORCM, Journal of Computational Science and Technology, Vol. 7 (2013), pp. 114-125. 